\title{
Anisotropic friction: assessment of force components and resulting trajectories
}

\author{
F. Tapia, D. Le Tourneau and J.-C. Géminard*
}

${ }^{*}$ Correspondence: jean-christophe.geminard@ens-lyon.fr 1 Laboratoire de Physique, Université de Lyon, Ecole Normale Supérieure de Lyon, CNRS, 46 Allée d'Italie, 69007 Lyon, France

\section{照 Springer}

\begin{abstract}
We report on an experimental device that makes it possible to assess the frictional properties of the contact between a slider and a horizontal surface, and to study the resulting trajectories of the slider when pulled across the surface by means of a flexible link. First, we show experimentally that, when the frictional properties are anisotropic, the slider is subjected, in addition to the dissipative frictional force oriented along the trajectory, to a force, perpendicular to the trajectory, which thus does not contribute to energy dissipation. Therefore, the slider does not necessarily moves in the pulling direction. Second, we show that the trajectories of the slider, when in continuous motion, in absence of inertial effects, can be recovered by assuming that, at all time, the friction force compensates the pulling force. We point out that we prove experimentally that the normal component of the friction force is given as the derivative, with respect to the sliding direction, of the tangential component. This result is particularly interesting as the relation between the normal and the tangential components is compatible with "the maximum of energy release rate" criterion used in the theory of fracture.
\end{abstract}

Keywords: Mechanical contact, Solid friction, Anisotropic friction

\section{Introduction}

Fractures are present widely in our daily life. Engineering and technical activities are often related to fracturing process, which involve dramatic events of large structure such as aircraft fuselages and silos as well as in controlled process, in which the ductility of bulk metallic glasses is improved by high localized micro-cracks [1]. Because of their practical importance, they have been of interest of engineers and scientists for decades [2, 3].

Regarding the fracturing process, there are several important questions, among them the initiation of the fracture and the fracture path. For isotropic materials, these questions have been considered using energetic and symmetry arguments. For anisotropic materials, however, simple symmetry arguments cannot be used. Indeed, concerning the path, the fracture does not necessarily propagate perpendicular to the direction of maximum opening stress. For example, fractures forced to propagate across a thin sheet of anisotropic brittle material exhibit striking oscillations, and even kinks, around the spiral path expected in anisotropic materials [4-6]. In order to predict the path of fractures in anisotropic materials, criteria were proposed, among them a "maximum energy release rate" criterion whose applicability still deserves to be tested [7].

(C) 2016 Tapia et al. licensee Springer on behalf of EPJ. This is an Open Access article distributed under the terms of the Creative Commons Attribution License (http://creativecommons.org/licenses/by/4.0), which permits unrestricted use, distribution, and reproduction in any medium, provided the original work is properly cited. 
When anisotropic materials are considered, it is interesting to distringuish the role played by the physical properties, considered independently from one another. For instance, both the elastic properties and the energy release associated to the opening of the fracture can be anisotropic. In order to consider the effect of the anisotropy of the energy release only, we propose to consider the analogy between fracture and friction. Indeed, the propagation of a fracture leads to the creation of two new surfaces and the associated energy release increases linearly with the fracture length, whereas elastic energy loaded in the material is released $[2,3,8]$. Similarly, when a slider is pulled by means of a spring across a flat substrate, the energy dissipated by friction increases linearly with the sliding distance, whereas elastic energy loaded in the spring is released [9]. Beyond a simple analogy, the study of the transition to sliding motion in friction shows that the phenomenon can be regarded as a fracturing process [10].

Drawing a parallel between fracture and friction, we thus propose to consider the trajectory of a solid body (a slider) pulled across a solid surface exhibiting anisotropic frictional properties. Interestingly, when the slider is pulled by means of an inextensible thread, the sliding is in continuous motion, which makes it possible to focus on the effects of the anisotropy of the dynamical frictional coefficient alone. The equivalent in fracture would be to consider the path of a crack that propagates, in absence of fits and starts, in a material exhibiting anisotropy of the fracturing energy.

Our aim is to focus on the trajectory of the slider. However, we must here mention previous results obtained concerning the anisotropic friction. At the microscopic scale, the possibility to measure the normal and the components of the force applied the AFM tip [11] made the latter an appropriate tool to study the friction at the atomic level [12]. In the case of an anisotropic surface, the tip is subjected to a transverse force which can be also measured by considering the torsion of the cantilever [13]. At the macroscopic scale, measurements of the frictional force showed that the "maximum energy release rate" criterion should apply but the two components of the force were not obtained indepently [14]. Another interesting effect of the transverse frictional force is the torque applied to a wheel pulled along its axis across the surface $[15,16]$.

In the first experimental configuration [17], we observed that, in the limit of small anisotropy, the paths of the slider forced to describe closed trajectories, are correctly predicted by using the criterion of "maximum of energy release rate". However, the study was limited to small anisotropy and the properties of the frictional contact, in particular the friction force, was not measured. Here we report on a novel experimental device which makes possible to characterize the frictional properties of the contact between the surface and the slider, by measuring directly the frictional force, and to observe the resulting trajectory of the slider.

\section{Experimental device}

The experimental device consists of a horizontal table at the upper surface of which a solid body, linked to the fixed point $\mathrm{P}$ in the frame of the laboratory by means a flexible thread of length $a$, is forced to slide (Fig. 1).

The table, a disk (Polyvinyl chloride, diameter $50 \mathrm{~cm}$ and thickness $1 \mathrm{~cm}$ ), is put in rotation, in the clockwise direction, around its center $\mathrm{O}$ by means of a wheel that rolls on its edge. The wheel is driven by a DC motor (Crouzet, 80807018 ) fed by a power supply 


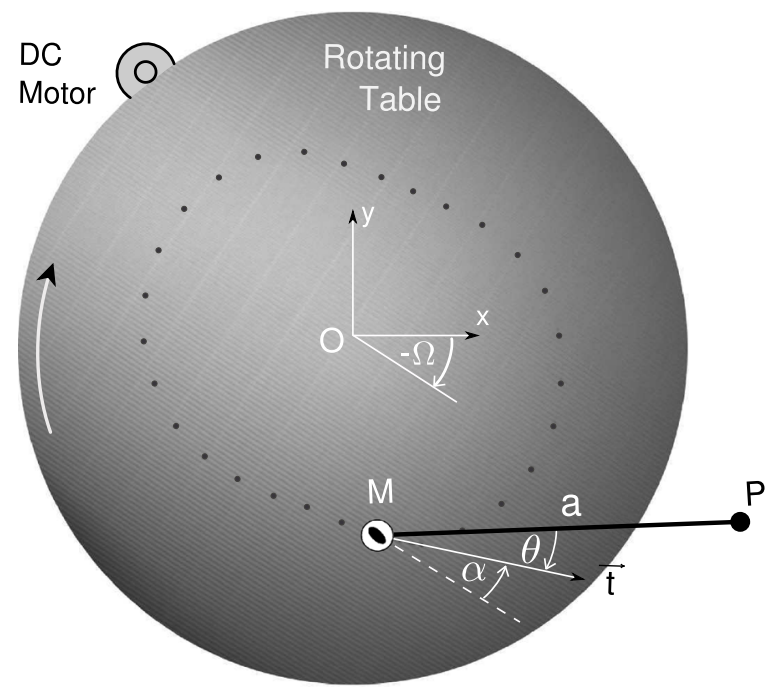

Fig. 1 Top view of the experimental device - The slider (M) is lying on top of a horizontal circular table. The slider is attached to a fixed point $\mathrm{P}(\mathrm{MP} \equiv a$ ) while the table is rotated (angular position $\Omega$ in the $x y$ frame of the laboratory) thanks to a DC motor. As a consequence of the anisotropy of the substrate, the tangent to the trajectory $\mathbf{t}$ makes the angle $\theta$ with the direction MP of the applied force. We denote $\alpha$ the angle that $\mathbf{t}$ makes with an axis attached to the table. We show here a photograph of the table surface, the black dots corresponding to successive positions of the slider marked with a pen

(Agilent, E3641A). The resulting rotation speed, $\omega$, ranges from 1-14 rpm. The angular position of the table $\Omega=\omega t$ is measured, with an accuracy of $1 \mathrm{deg}$, thanks to a coupled diode-photodiode system which locates black and white stripes drawn on the lower surface of the table.

The slider is a brass disk (thickness $1 \mathrm{~cm}$ and diameter $4 \mathrm{~cm}$ ) and the link is a nylon fishing line of diameter $270 \mu \mathrm{m}$. At one end, in order to ensure that it does not exert any significant torque, the thread makes a loose loop around a lug located at the center $M$ of the top surface of the slider. At the other end, the thread is firmly attached to a point $\mathrm{P}$ of a rigid arm or of a force sensor, depending on the experimental configuration. The heightto-diameter aspect-ratio of the slider insures that the latter has little tendency to flip. In addition, the link is flexible but, due to the small weight of the slider $(m g \simeq 1.1 \mathrm{~N})$, almost inextensible in our experimental conditions. In principle, the distance $R=\mathrm{OP}$ and the length $a \equiv$ PM of the thread can be chosen arbitrarily large. In practice, they range from $0-30 \mathrm{~cm}$.

The position of the slider, i.e. the position of the point $M$ in the frame of the laboratory, is obtained by imaging the system from above by means of a video camera (DALSA, Falcon $1 \mathrm{M} 120 \mathrm{HG}$ ). The frame rate if of 10 frames per second and, the image field being of about $(25 \times 25) \mathrm{cm}^{2}$, a spatial resolution of $25 \mu \mathrm{m}$ is achieved. Associated with the measurement of the angular position of the table, the assessment of the position of $\mathrm{M}$ makes it possible to determine the trajectory of the slider in a frame of reference attached to the surface in rotation.

When necessary, two components of the driving force can be simultaneously measured by means of two sensors (Testwell, KD40S, nominal force $2 \mathrm{~N}$ ) connected to two multimeters (Agilent, 34410A). The location of the sensors, which depends on the chosen configuration, will be discussed along with the experimental results. 
The whole experimental setup is automatized (National Instruments, LabView 2011). Images are processed subsequently (MathWorks, Matlab R2012a). This improved (with respect to the one described in [17]) experimental device, is better in several ways. First, it makes it possible to achieve a better spatial resolution in the slider position because the field of view can be limited to the region explored by the slider, whereas imaging the whole table was previously necessary. However, the price we pay is that we do not observe directly the trajectory of the slider on the table. It has to be reconstructed from the angular position of the table and from the position of the slider in the laboratory frame of reference. Second, the setup makes it possible to measure forces, which was not previously the case.

In the following, we discuss data obtained in various experimental conditions, which will be detailed in the corresponding sections. However, we mention here that we consider only regimes of continuous sliding which involve a dynamical friction coefficient, only. Taking in account that the dynamical frictional coefficient $\mu$ is almost independent of the sliding velocity, we can assume (and we checked experimentally) that the angular velocity of the table does not play any significant role. We thus report results obtained for a unique value of the angular velocity of the table, $\omega=2 \mathrm{rpm}$.

\section{Experimental results}

\section{Transient on an isotropic surface}

In a first experimental configuration, we consider a surface exhibiting isotropic frictional properties. To do so, both the lower surface of the slider and the top surface of the table are covered with a Bristol board.

We remind that the slider is pulled by means of a thread having the length $a$ attached to a fixed point $\mathrm{P}$ such that $\mathrm{OP} \equiv R$ (Fig. 1). From now on, we denote $\rho \equiv \mathrm{OM}$, the distance between the slider $M$ and the center of rotation $O$ which might depend on $\Omega$. However, for an isotropic surface, by symmetry, the asymptotic trajectory is a circle. The additional condition that the velocity of the slider, relative to the table surface, is aligned with the pulling force, thus with PM, imposes the asymptotic radius $\rho_{0}=\sqrt{R^{2}-a^{2}}$.

In order to test our experimental device, we report on the transient during which the slider, initially at a distance $\rho \neq \rho_{0}$ reaches the asymtotic distance $\rho_{0}$. Let us assume that the slider is initially at a distance $\rho_{0}+\delta \rho$ from the center $\mathrm{O}$, the thread being however under tension. Taking into account that the velocity of the slider, relative to the table, remains aligned with PM, one can easily demonstrate [17], provided that $\delta \rho \ll \rho_{0}$, that the trajectory of the slider obeys:

$$
\rho(\Omega)=\rho_{0}+\delta \rho \exp \left(-\Omega / \Omega_{c}\right)
$$

with $\Omega_{c}=a / \rho_{0}=l / \sqrt{1-l^{2}}$ where $l \equiv a / R$ denotes the dimensionless length of the thread. Eq. (1) predicts that, for a surface exhibiting isotropic frictional properties, the asymptotic trajectory is a circle of radius $\rho_{0}=\sqrt{R^{2}-a^{2}}$ reached after a transient over the characteristic angular distance $\Omega_{c}=a / \rho_{0}$. We observe in Fig. 2 a quantitative agreement between the experimental and theoretical values of $\Omega_{c}$, which validates the experimental technique by means of a first example. 


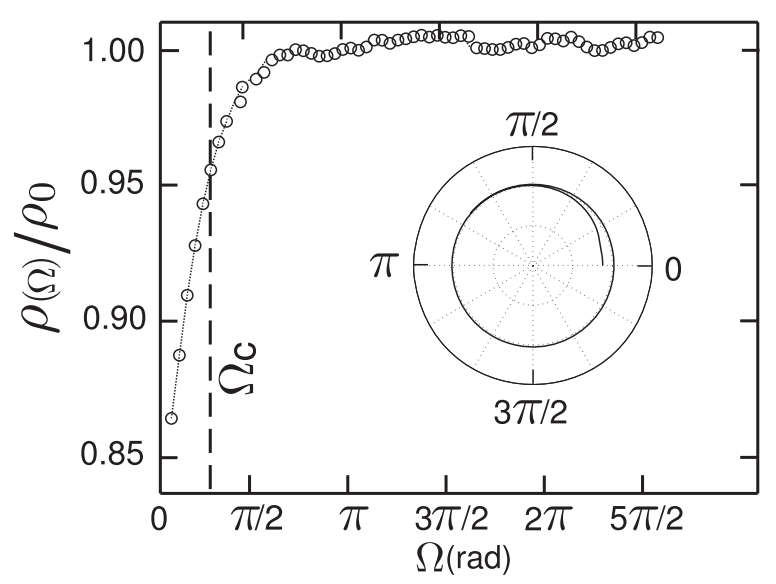

Fig. 2 Radius $\rho$ vs. angular position $\Omega$ - We observe that the asymptotic trajectory is reached after a typical rotation of about $\Omega_{c}=a / \rho_{0} \simeq 0.7 \mathrm{rad}$ in our experimental conditions. Inset: trajectory of the slider in the table frame (continuous line) [isotropic friction, $a=12.3 \mathrm{~cm}$ and $R=22.1 \mathrm{~cm}$ ]

\section{Anisotropic surface}

We now consider the case of an anisotropic sliding contact between the table and the slider.

We remind that, in the case of an anisotropic frictional contact, the friction force $\mathbf{F}$ is not necessarily aligned with the sliding direction. Thus, the force $\mathbf{F}$ is composed of two, qualitatively different, components. The first component, $\mathbf{F}_{t}$, is aligned with the sliding direction. This tangential component is opposed to the slider motion and its magnitude $F_{t}=\mu m g$, where $\mu$ is, by definition, the dynamical friction coefficient. For an isotropic frictional contact, there is no additional component of the friction force and $\mathbf{F}=\mathbf{F}_{t}$. However, if $\mu$ depends on the sliding direction (characterized here by the angle $\alpha$ that the velocity of slider makes with a reference direction of the substrate), the slider does not necessarily move in the pulling direction. Thus, the friction force is not aligned with the sliding direction, which implies that a normal component $\mathbf{F}_{n}$ (perpendicular to the sliding direction) is added to $\mathbf{F}$.

Let consider that the trajectory makes the angle $\theta$ with the applied force $\mathbf{F}$. For a displacement $\delta l$ of the slider along the trajectory (thus, in the direction of the tangent $\mathbf{t}$ ), the work of the friction force is $\delta \mathcal{W}_{\mu}=\mu m g \delta l$, whereas the work of the external force is $\delta \mathcal{W}=F \cos \theta \delta l$. In absence of elastic contribution (the link is assumed to be inextensible), the energy balance imposes $F \cos \theta=\mu m g$. The external force $F$ and $\theta$ being independent, we miss a relation to determine the sliding direction. In order to go farther, it is proposed to consider that the angle $\theta$ is the one maximizing the energy release rate, $\mathcal{E} \equiv-\left.\frac{\partial}{\partial l}\right|_{F}\left(\mathcal{W}-\mathcal{W}_{\mu}\right)$ at constant external force $F$. We have $\mathcal{E}=\mu m g-F \cos \theta$ and, thus, the criterion of "maximum of energy release rate" provides the additional condition $\left.\frac{\partial \mathcal{E}}{\partial \theta}\right|_{F}=\mu^{\prime} m g+F \sin \theta$ (the prime denotes the derivative with respect to the angle). Taking the energy balance into account, we finally get that the tangent to the trajectory $\mathbf{t}$ makes the angle $\theta$, such that $\tan \theta=-\mu^{\prime} / \mu$, with the applied force $\mathbf{F}$ (with MP in Fig. 1). Measuring the components of the force, we should measure $F_{n}=F_{t}^{\prime}$ [17].

Our aim is here to use our experimental setup to characterize the properties of the frictional contact by directly measuring the friction force and to observe the resulting trajectories of the slider. In order to achieve a friction force which exhibits a rather large, 
2-fold, anisotropy, the table is covered with a sheet of corrugated cardboard (wavelength $3.5 \mathrm{~mm}$, amplitude $1 \mathrm{~mm}$ ) whereas the lower surface of the slider is covered with a disk of lightweight felt (thickness about $1 \mathrm{~mm}$ ). Note that the corrugation of the surface is here only used a trick to introduce anisotropy of the frictional properties [18-20]. There is a vast literature concerning the relation between the physical properties of the surfaces in regard and the frictional properties, from both experimental and theoretical points of view [21]. As the question is out of the scope of the present study, we did not characterize further the corrugated surface. However, in Section "Force measurements", we thoroughly characterize the friction force, whereas the associated trajectory is discussed in Section "F-plot and associated trajectory".

\section{Force measurements}

In order to measure simultaneously the two components $F_{n}$ and $F_{t}$ of the friction force F, we use two force sensors in the configuration sketched in Fig. 3, top : the two sensors, that are in a plane parallel to the table surface, are perpendicular one to another. Special attention is paid to place the axis of one sensor in the radial direction (its axis points towards the center of rotation $O$ ), so that we indeed measure the radial component of $\mathbf{F}$, i.e. $F_{n}$. In this configuration, the second sensor measures the ortho-radial component of the force, i.e. $F_{t}$. We point out that, doing so, we impose that the slider is fixed in the frame of the laboratory and, thus, moves along a circle on the table.

In our experimental configuration, for a clockwise rotation, the slider is continuously pushed against the ortho-radial sensor. But, in constrast, the radial component of the force can push the slider against the sensor or pull it away from the sensor depending on the angular position of the table. In order to avoid the loss of contact, the slider is attached to the sensor by means of a flexible link. The link thus makes it possible to continuously measure the radial component of the force $F_{n}$ without introducing any significant bias in the measurement of both components. We checked that, provided the sensors are indeed perpendicular to one another and that one of them points toward the center of rotation, we measure accurately the two components $F_{n}$ and $F_{t}$ without any significant crosstalk. To do so, we compared the results obtained in the latter configuration to independent measurements of $F_{t}$ and $F_{n}$ obtained in two additional experimental configurations: $F_{t}$ by

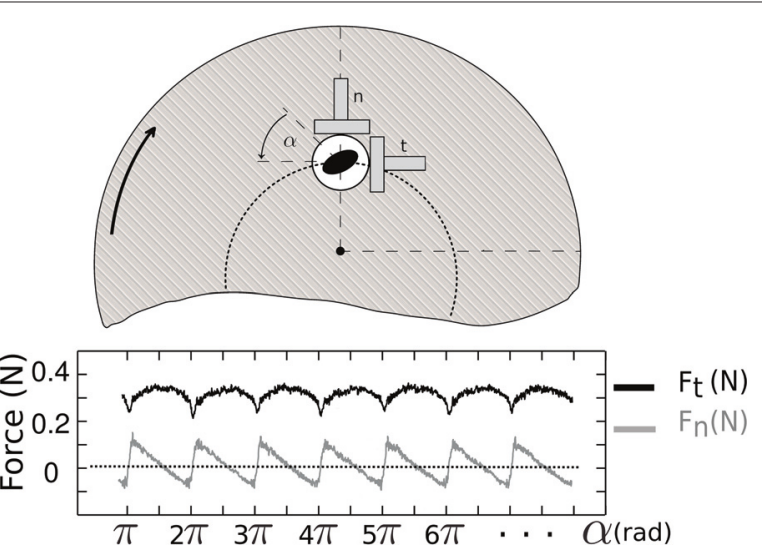

Fig. 3 Direct measurement of the two components of the friction force $\mathbf{F}$ - Top: Sketch of the experimental configuration. Bottom: Magnitude of the tangential $F_{t}$ and normal $F_{n}$ components of $\mathbf{F}$ as function of the sliding direction, $\alpha$ 
pulling the slider with a thread as described in Section "Transient on an isotropic surface" and measuring the pulling force at the fixed point (The estimation of $F_{t}$ then requires the knowledge of the position of the slider and some geometrical corrections); $F_{n}$ by pulling the slider with a long thread and measuring the force applied to the sensor pointing toward the center of rotation while it is in contact with it, during a half revolution. The agreement between the measurements indicate that there is no crosstalk between the sensors. We shall see that, however, there remains a defect in the measurement technique when the direction of easy sliding is approached. Because we use a flexible, loose, link, the slider tends to move slightly, and especially spin, when the velocity aligns with the corrugations. The data obtained in this region must be considered with caution.

In Fig. 3 bottom, we report the magnitudes $F_{t}$ (tangent to the sliding direction) and $F_{n}$ (normal to the sliding direction) as function of the sliding direction $\alpha$ for several table revolutions (We point out that the variation of the sliding direction $d \alpha$ and the variation of the angular position of the table $d \Omega$ have opposite signs, such that $d \alpha=-d \Omega$ ). We first note, from $F_{t}$, that the anisotropy is rather large, the friction coefficient $\mu$ oscillating between 0.22 and 0.3. Moreover, $\mu$ exhibits a complex pattern as a function of $\alpha$ that cannot be accounted for by a simple sinusoidal variation. Interestingly, we observe that $F_{n}$ is non zero, oscillates exhibiting also a complex pattern, but is zero in average. We remind here that the variation of $F$ are not due to any variation to the sliding velocity which remains constant in our experimental conditions, but only to the dependence of the frictional properties on the sliding direction $\alpha$. Note that the complex behaviour for $\mu(\alpha)$ was expected for the choice of a corrugated surface exhibiting a large anisotropy, in particular the rapid variation of $F_{t}$ around the direction of the corrugations as the sliding in this specific direction is significantly easier than in any other direction. We made that choice on purpose in order to test the validity of the relation between the normal and tangential components of the friction force beyond the, first order, sinusoidal variation of the frictional properties with the sliding direction, which we had already tested in [17].

In order to verify if $F_{n}$ is indeed the derivative of $F_{t}$ with respect to the sliding direction, we display, in Fig. 4, together $\int^{\alpha} F_{n} d \alpha$ and $F_{t}$ (Integrating $F_{n}$ avoids differentiating experimental data for $F_{t}$ and, thus, introducing undesired noise). We observe that the data superimpose, excepted in a narrow range of angle around the direction of minimum friction coefficient (i.e. $\alpha \simeq 0$ and $\alpha \simeq \pi$ ). The discrepancy is likely due to the defect in the experimental technique that we commented above. In spite of this small discrepancy, the agreement between both quantities is the direct experimental proof that, indeed, $F_{n}(\alpha)=F_{t}^{\prime}(\alpha)$ and, thus, that the "maximum of energy release rate" criterion applies.

\section{F-plot and associated trajectory}

Once the frictional force is characterized, we use the experimental device to study the resulting trajectories. As an example, we consider the experimental configuration depicted in Fig. 1 for the frictional contact characterized above. We report in Fig. 5, the trajectory of the slider in the frame attached to the table for $a=12.6 \mathrm{~cm}$ and $R=19.3 \mathrm{~cm}$, together with the polar-plot of the friction force $\mathbf{F}_{t}$. We observe that the trajectory significantly departs from a circle.

The trajectory can be accounted for as follows. In absence of inertial contribution, the friction force compensates at all times the applied force, which imposes that PM and $\mathbf{F}$ are collinear. Note, thus, that the geometry of the trajectory is not governed by the 


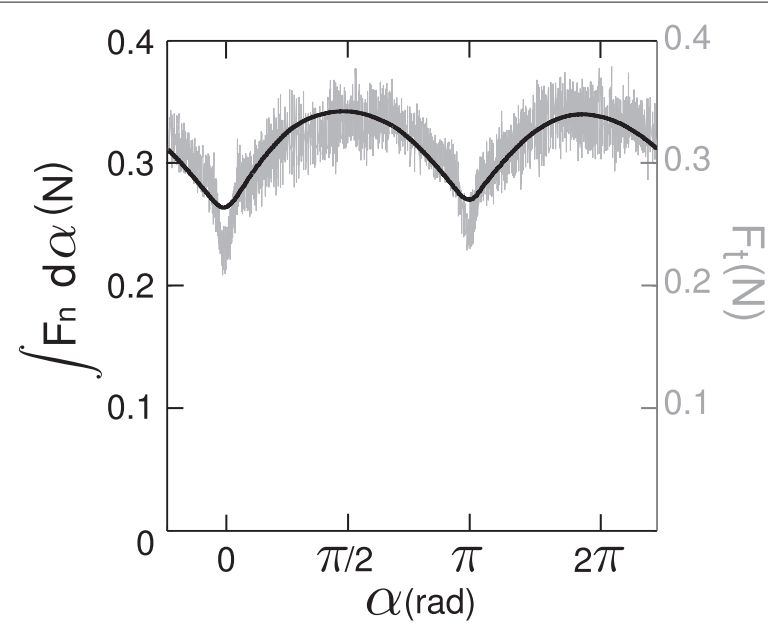

Fig. 4 Tangential component $F_{t}$ and primitive $\int F_{t} d \alpha$ vs. $\alpha$ - The overlap proves that, indeed, $F_{n}(\alpha)=F_{t}^{\prime}(\alpha)$

frictional properties alone, as it would be the case for the equilibrium shape of a crystal for instance [22-24], but that the geometry of the driving also comes into play. To go farther, we use an interpolation of the friction force in terms of $\cos (2 \alpha)$ and determine numerically (Wolfram, Mathematica 9.0), taking into account the simple condition that $\mathbf{P M} \times \mathbf{F}=\mathbf{0}$, the resulting trajectory. We observe an excellent agreement between the experimental trajectory and the prediction obtained using the experimental friction force, which validates the force measurements.

\section{Discussion and conclusion}

We reported on an improved experimental device dedicated to the study of anisotropic friction. The device not only makes it possible to assess the motion of a solid body forced

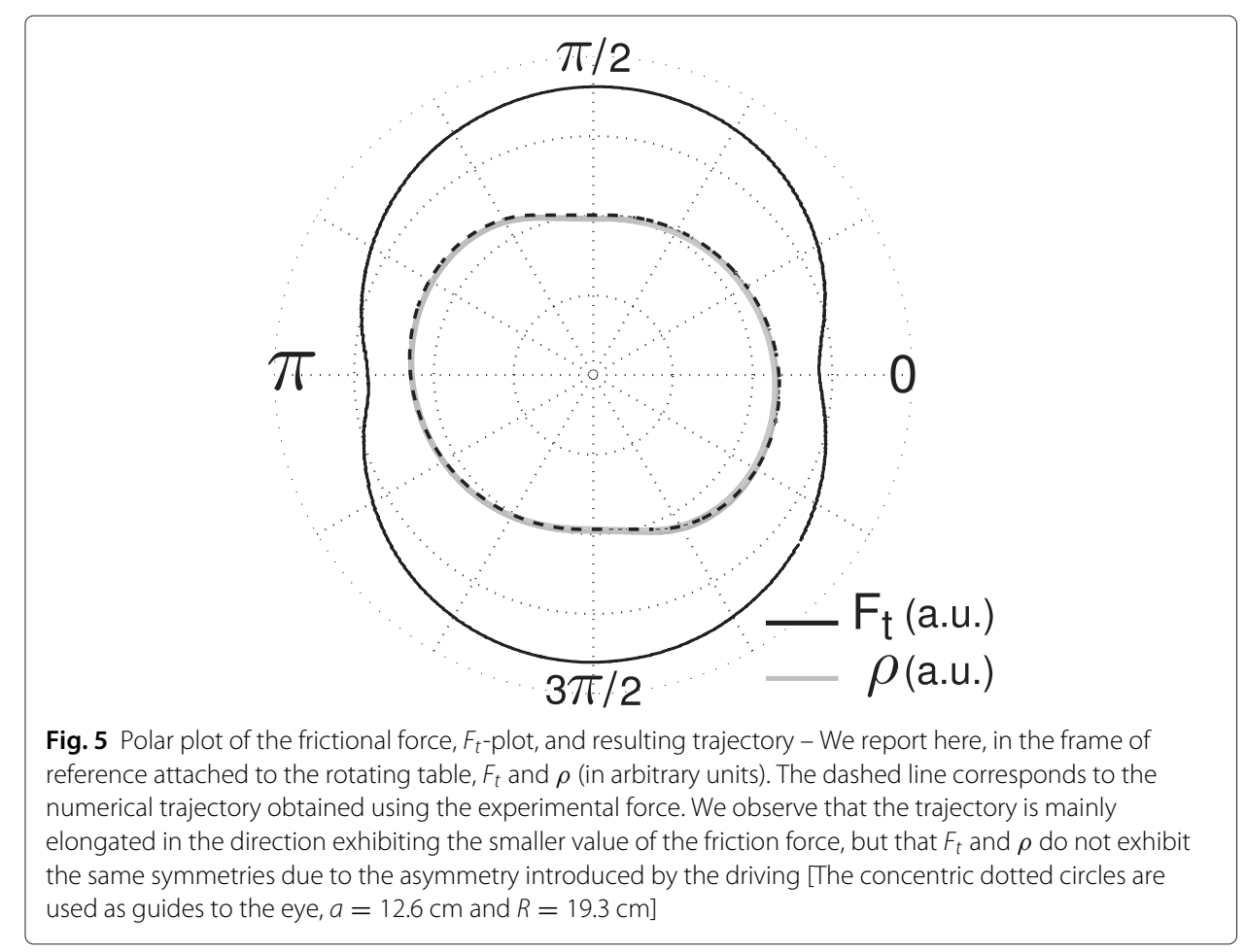


to slide along closed trajectories across an anisotropic surface but also to directly characterize the frictional force. We made use of the device to measure independently the two components of the frictional force, one tangent to the slider velocity which thus contributes to dissipation, the other perpendicular to the slider velocity which thus does not contribute to dissipation. Moreover, we showed that the perpendicular component is indeed the derivative, with respect to the sliding direction, of the tangential component, even when the anisotropy is large and exhibits a complex pattern beyong the sinusoidal modulation studied previously. Finally, having characterized the frictional force, we determined the resulting slider trajectory in a specific geometry of the driving and observed an excellent agreement with the theoretical trajectory based on the expression of the friction force. As is, these results nicely complement our former study of the problem [17] which was limited to small anisotropy and wich was missing forc measurements.

Coming back to analogy with fracture, we remind that we considered here the case of a slider in continuous motion whose dynamics can be accounted for by taking into account a unique frictional coefficient, independent of the velocity, which however can depend on the direction. The problem is the analogue of the quasistatic propagation of a fracture whose energy depends on the direction, but not on the velocity. Thus, the frictional system mimics the fracturing process in an anisotropic, brittle material. The experimental observation of a good agreement between the experimental trajectory and, even better, the direct experimental observation that the normal component is the derivative, with respect to the sliding direction, of the tangential component of the frictional force, prove that the criterion of the "maximum of energy release rate" can be used in this case and, thus, in the case of the brittle fracture.

This study deserves to be extended in several directions. On the one hand, we plan to study the effect of the increase of the anisotropy in order to observe if the trajectory can exhibit kinks or facets, as observed in crystals [24] or cracks [4, 5]. On the other hand, rapid changes in direction are likely to be associated with inertial effects, which can be by themselves the subject of a specific study. Thus, we are planning to introduce inertial effect by the use of an elastic thread. In this case, one expects to observe a stickslip motion, which involves inertial effects during the rapid slip events. What can we say of the criterion of "maximum of energy release rate" in this case?

Competing interests

The authors declare that they have no competing interests.

\section{Authors' contributions}

D Le Tourneau machined the experimental device. F Tapia performed the experiments under the supervision of J-C Géminard. FT and JCG analized the results and wrote the manuscript.

Acknowledgements

This work was funded by a post-doctoral grant of the CONICYT (contract number POSTDOCTORADO-2013 74140057).

Received: 23 October 2015 Accepted: 14 January 2016

Published online: 08 February 2016

\section{References}

1. Das J, Tang MB, Kim KB, Theissmann R, Baier F, Wang WH, Eckert J (2005) "Work-Hardenable" ductile bulk metallic glass. Phys Rev Lett 94(20):205501

2. Lawn BR, Wilshaw TR (1993) Fracture of Brittle Solids. Cambridge University Press, New York

Leblond J (2003) Mécanique de la rupture fragile et ductile. Hermes Science pulication - Lavoisier, Paris

Roman B (2013) Fracture path in brittle thin sheet: a unifying review on tearing. Int J Fract 182:209-237

Romero V, Roman B, Hamm E, Cerda E (2013) Spiral tearing on thin films. Soft Matt 9:8282-8288

6. Takei A, Roman B, Bico J, Hamm E, Melo F (2013) Forbidden directions for the fracture of thin anisotropic sheets: an analogy with the wulff plot. Phys Rev Lett 110:144301 
7. Francfort F, Marigo J-J (1998) Revisiting brittle fracture as a energy minimization problem. J Mech Phys Solids 46:1319

8. Griffith A (1920) The phenomena of rupture and flow in solids. Phil Trans Roy Soc London, CCXXI-A

9. Persson BNJ (1998) Sliding Friction: Physical Principles and Applications. Springer, New York

10. Rubinstein SM, Cohen G, Fineberg J (2004) Detachment fronts and the onset of dynamic friction. Nature 430:1005-1009

11. Meyer G, Amer NM (1990) Simultaneous Measurement of Lateral and Normal Forces with an Optical-Beam-Deflection Atomic Force Microscopy. Appl Phys Lett 57:2089-2091

12. Ogletree DF, Carpick RW, Salmeron M (1996) Calibration od frictional forces in atomic frce microscopy. Rev Sci Instrum 67:3298-3306

13. Kalihari V, Tadmor E, Haugstad G, Frisbie C (2008) Grain orientation mapping of polycrystalline organic semiconductor films by transverse shear microscopy. Adv Mater 20:1-7

14. Konyukhov A, Vielsack P, Schweizerhof K (2008) On coupled models of anisotropic contact surfaces and their experimental validation. Wear 264:579-588

15. Goyal S, Ruina A, Papadopoulos J (1991) Planar sliding with dry friction. Part 1. Limit surface and moment function. Wear 143:307-330

16. Goyal S, Ruina A, Papadopoulos J (1991) Planar sliding with dry friction. Part 2. Dymanics of motion. Wear 143:331-352

17. Chateau D, Géminard J-C (2013) Fracture path in an anisotropic material in the light of a friction experiment. Phys Rev E 88:033202

18. Carpick RW (2006) Controlling friction. Science 313:184-185

19. Zmitrowicz A (2006) Models of kinematics dependent anisotropic and heterogeneous friction. Int J Sol and Struct 43:4407-4451

20. Singh R, Melkote SN, Hashimoto F (2005) Frictional response of precision finished surfaces in pure sliding. Wear 258:1500-1509

21. Vanossi A, Manini N, Urbakh M, Zapperi S, Tosatti E (2013) Colloquium: Modeling friction: From nanoscale to mesoscale. Rev Mod Phys 85:529-552

22. Wulff GZ (1901) Kristallogr. Mineral 34:449

23. Woodruff DP (1973) The solid-liquid interface. University Press, Cambridge

24. Tiller WA (1991) The science of crystallization. University Press, Cambridge

\section{Submit your manuscript to a SpringerOpen ${ }^{\odot}$ journal and benefit from:}

- Convenient online submission

- Rigorous peer review

- Immediate publication on acceptance

- Open access: articles freely available online

- High visibility within the field

- Retaining the copyright to your article

Submit your next manuscript at $\boldsymbol{\wedge}$ springeropen.com 